Wird der Postwachstumsansatz von rechts vereinnahmt?

\section{Natürliche Feinde oder heimliche Verbündete?}

\author{
Immer wieder wird behauptet, die Postwachstumsdebatte \\ sei anschlussfähig gegenüber völkischem Denken oder anfällig \\ für eine Unterwanderung von rechts. Welche Argumente \\ werden dabei vereinnahmt und wie kann Wachstumskritik \\ unabhängig bleiben? Von Dennis Eversberg
}

M anche Kritiker/innen unterstellen der Postwachstumsdebatte, sie sei anfällig oder offen für eine Vereinnahmung oder Unterwanderung durch nationalistische und völkisch-rassistische Kräfte. Das scheint zunächst auch nicht ganz abwegig, weil beides Reaktionen auf die gleiche verunsichernde Weltlage sind und beides mit Kritik am globalisierten Kapitalismus einhergeht. Auch wenn solche pauschalen Unterstellungen auf unzulässigen Vereinfachungen beruhen, sind Befürworter/innen von Postwachstum und Degrowth gut beraten sich zu fragen, wo ihre Argumente mögliche Anknüpfungspunkte für eine solche Vereinnahmung bieten und was sie tun können, um diese unmöglich zu machen.

\section{Rechtspopulismus und Wachstum}

Besondere Aufmerksamkeit erhält derzeit wegen seiner politischen Erfolge der Rechtspopulismus. Als politische Strategie beruht dieser auf der Behauptung, man stehe für das „wahre Volk“, das von anderen Parteien nicht mehr angemessen vertreten werde, und greift verbreitete, oft widersprüchliche alltägliche Denkmuster und Vorurteile auf, um Wahlerfolge zu erzielen.

Mit Wachstumskritik können Kräfte, die einer solchen politischen Logik folgen, meist überhaupt nichts anfangen, weil Wachstum für das „gesunde Volksempfinden“, an dem sie sich ausrichten, ein unhinterfragt positiver Wert ist. Typisch ist eine Äußerung der Spitzenkan- didatin der Alternative für Deutschland (AfD), Alice Weidel, vom 1.6.2017:

„Schäuble und Merkel stellen sich gerne als Wachstumsgeneratoren, Exportweltmeister und Arbeitsplatzbeschaffer dar. [...] Angesichts [ihrer] politischen Fehlleistungen ist es eine Narrenposse, wenn sich Schäuble und Merkel des wirtschaftlichen Aufschwungs brüsten und Schäuble gar behauptet, er

Kräfte wie die AfD leben also nicht zuletzt davon, dass sie der Mehrheit „Wachstum“, sprich weiter steigenden Wohlstand, versprechen. Deshalb ist es derzeit kaum denkbar, dass sie sich der Wachstumskritik zuwenden könnten.

\section{Völkischer Rassismus: Wachstumskritik von rechts}

Eine solche populistische Politik muss aber unterschieden werden von offen völkisch-rassistischen und neonationalsozialistischen Ideologien. Deren Ziele sind weit weniger beliebig. All ihre Spielarten beruhen auf der Behauptung einer „naturgegebenen“ Ungleichwertigkeit von Menschen, und sie gehen auf bestimmte historische Traditionslinien zurück. Neben dem Nationalsozialismus und anderen faschistischen Bewegungen zählt hierzu auch die Strömung der sogenannten "Konservativen Revolution“ in der Zwischenkriegszeit. Auf letztere berufen sich Autor/innen und Gruppierungen der "Neuen Rechten“ wie der Verleger Götz Kubitschek und die betont jugendlich-popkulturell auftretende „Identitäre stünde für Wachstum.“
Bewegung“. Die „Neue Rechte“ steht für den Versuch einer „intellektuellen“ Neubegründung völkischen Denkens. In Abgrenzung vom biologistischen Rassismus des Nationalsozialismus vertritt sie die bis in die politische Mitte hinein verbreitete Vorstellung, dass Ungleichheiten zwischen Menschen wegen ihrer Zugehörigkeit zu historisch gewachsenen Kulturen berechtigt und eine Vermischung dieser Kulturen schädlich sei. Die damit verbundene Vorstellung des „Ethnopluralismus" besagt, dass alle "Völker" ihr Existenzrecht hätten, solange sie da blieben, wo sie hingehörten. Auch die „Neue Rechte" teilt aber den Kernglaubenssatz des völkischen Rassismus, dass es verschiedene Menschentypen gebe, die unterschiedlich und ungleich seien und es zwingend bleiben müssten. Damit einher geht immer die Berufung auf „Identität" und "das Eigene“, das zu schützen und zu erhalten sei. Im Kern ist völkisch-rassistisches Denken stets anti-egalitär, anti-universalistisch und anti-individualistisch. Wachstum wird von diesen Ideologien kritisch gesehen, weil es als Begleiterscheinung der Globalisierung und der Zunahme der Weltbevölkerung eine Bedrohung für die eigene ethnische Reinheit und nationale Identität darstelle.

Nun finden sich aber auch in populistischen Gruppierungen nicht wenige Einzelpersonen oder ganze Strömungen, die einer völkisch-rassistischen Ideologie anhängen und die Orientierung am schnellen Wahlerfolg eher aus machttaktischen Gründen mittragen. Das bekannteste Beispiel in der AfD ist der thüringische Landesvorsitzende Björn Höcke, der seine Vorstellungen einer völkischen Wende mit expliziter Wachstumskritik verbindet. 2014 sagte er auf einer Feier der AfD-Jugend:

„Ich meine, dass wir Mitte des 21. Jahrhunderts an die Grenzen der Tragfähigkeit unseres Planeten angelangt sein werden. [...] Wir müssen überlegen, wie eine Postwachstumsökonomie aussieht. [...] Wir müssen eine Art von Wirtschaft entwickeln, die Ökologie und Ökonomie miteinander versöhnt, und das geht nur, wenn wir diese Art von Kapitalismus überwinden." 
Im Kern richtet sich diese Kritik aber nicht auf die Steigerungs- und Expansionszwänge des kapitalistischen Wettbewerbsprinzips, sondern auf den Zins:

„Die gegenwärtige Krise ist definitiv keine [...] der Marktwirtschaft, sondern eine [...] des zinsbasierten Kapitalismus. Wenn es [...] nicht bald gelingt, ein Geldsystem zu erschaffen, das nicht darauf angewiesen ist, ein ewiges Wachstum in einer endlichen Welt zu generieren, wird die letzte Hoffnung auf eine Selbstregulation von Mensch und Natur aufgegeben werden müssen. " (Leserbrief an die Junge Freiheit 2008)

Damit stellt er sich in eine lange Tradition rechter „Kapitalismuskritik“, die das Problem nicht in Wettbewerb und Profit, sondern in antisemitisch aufgeladener Weise im „parasitären“ Wesen des verzinslichen Kredits ausmachen.

Höcke ist aber nicht der einzige am rechten Rand, der sich wachstumskritischer Argumente bedient. Auch der französische neurechte Vordenker Alain de Benoist sorgte 2009 mit einem Buch für Aufsehen, in dem er sich wachstumskritische Forderungen wie Serge Latouches Ruf nach einer „Dekolonialisierung des Vorstellungsraums“ zu eigen machte. Und auch die neurechte Szene in Deutschland interessiert sich für Wachstumskritik. So veröffentlichte Felix Menzel, Stammautor des von Götz Kubitschek herausgegebenen Debattenblatts „Sezession“ und selbsterklärter intellektueller Kopf der „Identitären Bewegung“, im Blog der „Sezession“ eine elfteilige Reihe zur Wachstumskritik, in der er diverse Bücher (auch links verorteter Autor/innen) diskutiert und gestützt auf deren Argumente eine rechte Postwachstumsvision ausformuliert. Kernpunkt bei ihm ist, neben der wie bei Höcke zentralen Zinskritik, das Bevölkerungswachstum im globalen Süden, in dem Menzel eine Bedrohung für die europäischen Gesellschaften sieht. Um dieser zu begegnen, müsse in erster Linie Migration verhindert werden - ganz im Sinne des ethnopluralistischen Dogmas, dass alle bleiben sollen, wo sie „hingehören“. Dagegen fordert er eine Abkehr von der Globalisierung und eine Rückbesinnung auf das
„Eigene“, „Ursprüngliche“ und „Gewachsene" nationaler und regionaler Kulturen. Hier, so behauptet Menzel, sei man sich mit linken Kritiker/innen von Wachstum und Globalisierung einig:

„Einwanderungs-, Globalisierungsund Wachstumskritik gehören heute zusammen und müssen eine Metaideologie angreifen, der sowohl Unternehmen als auch Staaten gehorchen. Diese Metaideologie fordert grenzenlose Flexibilität und will uns beweisen, dass ein bescheidenes Leben auf der Basis des gesunden Menschenverstandes nicht mehr möglich sein soll.“

Dahinter steht eine dichotome Sicht der weltpolitischen Situation, derzufolge sich zwei große Lager gegenüberstünden: Einerseits (Neo-)Liberale und globalistische Linke mit ihrer Wachstumsund Flexibilitätsideologie, und andererseits jene, die zurück wollen zu einem bescheiden-,,vernünftigen“ Leben ohne Wachstum, Globalisierung und Einwanderung. Hiermit verbindet sich für ihn aber untrennbar die völkische Ideologie, derzufolge die gegen das Globale in Stellung gebrachten kleineren Gemeinschaften zwingend durch gemeinsame $\mathrm{Ab}$ stammung und Kultur, also ethnisch-national, bestimmt sein müssten und sich nicht vermischen dürften.

\section{Wie unvereinnahmbar bleiben?}

Was also macht eine Postwachstumsposition aus, die gegen solche Vereinnahmungsversuche gefeit ist? Wenn sie sich in erster Linie als ökologische und gegen Phänomene sozialer Beschleunigung und Entfremdung gerichtete Bewegung versteht, läuft Wachstumskritik leicht Gefahr, in die Falle von Menzels falscher Dichotomie zu tappen und sich den falschen „Verbündeten“ zuzuwenden. Eine Position wie die vieler Degrowth-Befürworter/innen dagegen, die sich als Teil einer globalen Gerechtigkeitsbewegung begreifen und die Aufhebung wirtschaftlicher Steigerungszwänge als Voraussetzung ökologischer und sozialer Gleichheit aller Menschen verstehen, ist dagegen immun. Statt aus der Kritik des ökonomistischen Universalismus des Kapitals den falschen Schluss der Hinwendung $\mathrm{zu}$ essentialistischen, im Ergebnis rassistischen Vorstellungen „natürlicher Gemeinschaften“ zu ziehen, verortet sie sich in einem dritten Lager, dem eines auf der Forderung nach gleichen Rechten und Möglichkeiten für Alle beruhenden global-solidarischen Universalismus. Wenn sich der Protest gegen ökologische Zerstörung und ökonomistische Vereinseitigung menschlicher Beziehungen aus dieser Gleichheitsforderung ergibt, folgt daraus auch eine Kritik an der globalen Ungerechtigkeit der „imperialen Lebensweise“ (Brand/Wissen 2017) und der Abschottungspolitik kapitalistischer Zentren, eine Parteinahme gegen rassistische Anti-Einwanderungsbewegungen und ein Beharren auf der Universalität der Menschenrechte - also das glatte Gegenteil des völkisch-rassistischen Denkens.

Besonders stark in Gefahr einer Vereinnahmung sind dagegen Formen der Wachstumskritik, die in erster Linie Zins, Geldsystem oder Überbevölkerung für globale Probleme verantwortlich machen und die Lösung im Zurück zu einem angeblich besseren, nachhaltigeren $\mathrm{Zu}$ stand „ursprünglicher“ Gemeinschaften ausmachen. Das heißt nicht, dass Geldkritik, Gemeinschaft oder die Anzahl der Menschen auf der Welt in der Postwachstumsdebatte Tabuthemen sein sollten wohl aber, dass diese Fragen im Kontext eines Selbstverständnisses als globaler Gerechtigkeitsbewegung anders, vorsichtiger und weniger zentral zu stellen wären, als das heute mitunter der Fall ist.

\section{Literatur}

Brand, U./Wissen, M. (2017): Imperiale Lebensweise. Zur Ausbeutung von Mensch und Natur im globalen Kapitalismus. München, oekom

AUTOR + KONTAKT

Dr. Dennis Eversberg ist wissenschaftlicher Mitarbeiter in der DFG-Kollegforscher/ innengruppe „Postwachstumsgesellschaften“.

Friedrich-Schiller-Universität Jena, Institut für Soziologie, Humboldtstr. 34 07743 lena. Tel.: +49 3641 9-45039 E-Mail: dennis.eversberg@uni-jena.de 